\title{
Effectiveness in Switching from Antipsychotic Polypharmacy to Monotherapy in Patients with Schizophrenia: A Case Series
}

\author{
Hiroyuki Kamei ${ }^{1,3}$, Hanae Yamada ${ }^{3}$, Masakazu Hatano ${ }^{1,2,3}$, Manako Hanya ${ }^{3}$, Shigeki Yamada ${ }^{2}$, Nakao Iwata \\ Departments of ${ }^{1}$ Psychiatry and ${ }^{2}$ Clinical Pharmacy, Fujita Health University School of Medicine, Toyoake, ${ }^{3}$ Office of Clinical Pharmacy Practice \\ and Health Care Management, Faculty of Pharmacy, Meijo University, Nagoya, Japan
}

\begin{abstract}
In Japan, drug therapy for schizophrenia is characterized by high-dose antipsychotic polypharmacy, which is an uncommon approach internationally. In this study, we reduced the number of antipsychotic agents in 5 patients using the Safety Correction of High-dose Antipsychotic Polypharmacy (SCAP) method and conducted a survey regarding treatment satisfaction. The switch from polypharmacy to monotherapy was achieved in all patients. There was no deterioration in psychiatric symptoms, and adverse reactions were reduced. Three of the subjects were satisfied with the decrease in the number of antipsychotic agents and dose-reduction. These results suggest that the SCAP method is a safe and useful method that can be applied in a clinical setting.
\end{abstract}

KEY WORDS: Schizophrenia; Antipsychotic agents; Polypharmacy; Patient satisfaction.

\section{INTRODUCTION}

Antipsychotic polypharmacy is prevalent in clinical practice despite the lack of supporting evidence [1]. Antipsychotic polypharmacy raises concerns regarding increases in total dosages, side effects, and mortality as well as decreased adherence [2]. In Japan, drug therapy for schizophrenia is also characterized by high-dose antipsychotic polypharmacy. Currently, strategies to correct high-dose antipsychotic polypharmacy for patients with schizophrenia are being conducted $[3,4]$. However, the proportion of patients receiving polypharmacy remains high and leads to high-dose administration $[3,4]$. According to many prescription surveys in Japan, between $26 \%$ and $34 \%$ of patients with schizophrenia are treated with a single agent, whereas $32 \%$ and $42 \%$ of patients undergo therapy with more than 3 agents $[5,6]$. Monotherapy is recommended due to the following dis-

Received: May 24, 2018 / Revised: July 13, 2018 Accepted: July 31, 2018

Address for correspondence: Hiroyuki Kamei Office of Clinical Pharmacy Practice and Health Care Management, Faculty of Pharmacy, Meijo University, 150 Yagotoyama, Tenpaku, Nagoya, Aichi 468-8503, Japan E-mail: hkamei@meijo-u.ac.jp

ORCID: https://orcid.org/0000-0003-3778-2352 advantages of high-dose antipsychotic polypharmacy [7]: 1) difficulty in evaluating which drug is effective or ineffective, 2) difficulty in deciding the optimal dosage, 3) difficulty in identifying the causes of adverse reactions, 4) complex regimens that increases the frequency of administration mistakes, thus enhancing the stress of the patient's family or nursing staff, 5) drug interactions, and 6) reduced compliance. To overcome these challenges, a method for safely correcting high-dose antipsychotic polypharmacy (SCAP method) has been proposed $[8,9]$. In the SCAP method, it is recommended that when the titer of an antipsychotic agent is high, the dose be reduced by 0 to $50 \mathrm{mg} /$ week as a chlorpromazine (CP)-converted value, and that when the titer is low, the dose be reduced by 0 to $25 \mathrm{mg} /$ week as a CP-converted value $[8,9]$. These dose-reduction rates have been designed to avoid the withdrawal symptoms mediated by dopaminergic $D_{2}$, cholinergic or other receptors.

In the present study, we reduced the number and doses of antipsychotic agents (dose-reduction) in patients with schizophrenia using the SCAP method $[8,9]$ and also reduced the dosages of antiparkinson and benzodiazepine drugs. In addition, we evaluated psychiatric symptoms and adverse reactions of patients in whom the number and doses of antipsychotic agents were reduced and con-

(ㄷ) This is an Open-Access article distributed under the terms of the Creative Commons Attribution Non-Commercial License (http://creativecommons.org/licenses/by-nc/4.0) which permits unrestricted non-commercial use, distribution, and reproduction in any medium, provided the original work is properly cited. 
ducted a survey regarding their satisfaction.

\section{CASE}

A study was conducted in 5 patients with schizophrenia who had consulted the outpatient clinic of the Department of Psychiatry, Fujita Health University Hospital between December 2013 and June 2014. Five patients had been taking two or more antipsychotic agents (continuously, for three months or more) with a total CP-converted dose of $600 \mathrm{mg}$ or higher. Informed consent was obtained from each patient. The study was approved by the ethics committee of the hospital.

According to the SCAP protocol [9], the dosage of one antipsychotic agent was reduced every week for the subjects in the dose-reduction group. The choice of agent to be reduced in dose was left to the discretion of the physician. High-potency antipsychotics are defined as agents for which a dose equivalent of $100 \mathrm{mg}$ of $\mathrm{CP}$ is achieved with $10 \mathrm{mg}$ or less, whereas agents for which the dose equivalent of $\mathrm{CP}$ exceeds $10 \mathrm{mg}$ are considered low-potency. Agents with low-potency were reduced by 0 to $25 \mathrm{mg} /$ week using the Japanese version of their CP dose equivalents, whereas dosages of high-potency agents were reduced by 0 to $50 \mathrm{mg} /$ week of their CP dose equivalents. Each week, the physician determined whether to reduce the dose or temporarily revert to a higher dose based on the patient's condition. Because the criteria for selecting monotherapy drugs has not been set, physicians selected the monotherapy drugs by themselves when switching from polypharmacy to monotherapy in this study.

Table 1. Changes in antipsychotic agents, antiparkinson and benzodiazepine drugs before and after dose-reduction

\begin{tabular}{|c|c|c|c|c|c|c|}
\hline \multirow{2}{*}{ Variable } & \multicolumn{6}{|c|}{ Patient No. } \\
\hline & 1 & 2 & 3 & 4 & 5 & Total \\
\hline \multicolumn{7}{|l|}{ Characteristic } \\
\hline Sex & M & M & M & $\mathrm{F}$ & $\mathrm{F}$ & \\
\hline Age $(y r)$ & 26 & 17 & 22 & 34 & 52 & $30.2 \pm 12.2$ \\
\hline Duration (yr) & 0.8 & 0.5 & 7 & 10 & 16 & $6.9 \pm 5.8$ \\
\hline \multicolumn{7}{|l|}{ Antipsychotic agent } \\
\hline \multicolumn{7}{|l|}{ Before dose-reduction } \\
\hline Anti-psychotic agent (mg) & $\begin{array}{c}\text { ARI, 18; SUL, } \\
\text { 100; RIS, } 1\end{array}$ & $\begin{array}{c}\mathrm{PAL}, 9 ; \mathrm{BLO} \\
4 ; \mathrm{LEV}, 5\end{array}$ & $\begin{array}{l}\text { BLO, 24; } \\
\text { CP, } 25\end{array}$ & $\begin{array}{l}\text { PAL, 3; } \\
\text { ARI, } 18\end{array}$ & $\begin{array}{l}\text { PAL, 9; } \\
\text { OLA, } 5\end{array}$ & $2.4 \pm 0.9^{*, \mathrm{a}}$ \\
\hline CP-converted dose (mg) & 600 & 800 & 625 & 650 & 800 & $695 \pm 97.5$ \\
\hline \multicolumn{7}{|l|}{ After dose-reduction } \\
\hline Anti-psychotic agent (mg) & ARI, 18 & QUE, 700 & BLO, 24 & ARI, 18 & PAL, 12 & $1.0 \pm 0.0^{*, a}$ \\
\hline CP-converted dose (mg) & 450 & 1,060 & 600 & 450 & 800 & $672 \pm 26$ \\
\hline \multicolumn{7}{|l|}{ Antiparkinson drug } \\
\hline \multicolumn{7}{|l|}{ Before dose-reduction } \\
\hline Anti-parkinson drug (mg) & TRI, 1 & $\mathrm{BP}, 2$ & $\mathrm{BP}, 3$ & $\mathrm{BP}, 6$ & $\mathrm{BP}, 3$ & $1.0 \pm 0.0^{\mathrm{a}}$ \\
\hline BP-converted dose (mg) & 0.5 & 2 & 3 & 6 & 3 & $2.9 \pm 2.0$ \\
\hline \multicolumn{7}{|l|}{ After dose-reduction } \\
\hline Anti-parkinson drug (mg) & - & - & $\mathrm{BP}, 3$ & BP, 6 & - & $0.6 \pm 0.5^{\mathrm{a}}$ \\
\hline BP-converted dose (mg) & - & - & 3 & 6 & - & $1.8 \pm 2.6$ \\
\hline \multicolumn{7}{|l|}{ Benzodiazepine drug } \\
\hline \multicolumn{7}{|l|}{ Before dose-reduction } \\
\hline BZP drug (mg) & LOR, 1.5 & LOR, 1 & FLU, 1; NIT, 15 & LOR, 1 & $\mathrm{BRO}, 0.25$ & $1.2 \pm 0.4^{\mathrm{a}}$ \\
\hline DAP-converted dose (mg) & 6.25 & 4.2 & 20 & 4.1 & 5 & $7.9 \pm 6.8$ \\
\hline \multicolumn{7}{|l|}{ After dose-reduction } \\
\hline BZP drug (mg) & - & - & FLU, $1 ;$ NIT, 15 & LOR, 1 & $\mathrm{BRO}, 0.25$ & $0.8 \pm 0.8^{\mathrm{a}}$ \\
\hline DAP-converted dose (mg) & - & - & 20 & 4.1 & 5 & $5.8 \pm 8.3$ \\
\hline
\end{tabular}

Values are presented as data only or mean \pm standard deviation.

M, male; F, female; ARI, aripiprazole; SUL, sulpiride; RIS, risperidone; PAL, paliperidone; BLO, blonanserin; LEV, levomepromazine; CP, chlorpromazine; OLA, olanzapine; QUE, quetiapine; TRI, trihexyphenidyl; BP, biperiden; BZP, benzodiazepine; LOR, lorazepam; FLU, flunitrazepam; NIT, nitrazepam; BRO, brotizolam; DAP, diazepam.

${ }^{*} p<0.05$ vs. before dose-reduction (paired $t$ test).

${ }^{a}$ Number of drugs. 
We used the Brief Psychiatric Rating Scale (BPRS) and the Drug-induced Extrapyramidal Symptoms Scale (DIEPSS) to assess psychiatric symptoms and extra-pyramidal side effects, respectively. We also assessed insight and adherence using the Japanese version of the Schedule for Assessment of Insight (SAI-J) and Drug Attitude Inventory-10 Questionnaire (DAI-10), respectively. These assessments were conducted prior to the start and one month after completion of the dose-reduction. The evaluation tools used here are standardized in Japan. In addition, a questionnaire for patients regarding their psychiatric symptoms and adverse reactions prior to the start and one month after the completion of the dose-reduction was conducted. Furthermore, their satisfaction with the treatment one month after the completion of the dose-reduction was assessed. The psychiatric symptoms and adverse reactions were subjectively evaluated ( 1 , absent; 2 , nonevaluable; 3, moderate; and 4, severe). The satisfaction with treatment was also subjectively evaluated (1, absent; 2, non-evaluable; 3, moderate; and 4, high). The scores used in this study were compared using paired $t$ test. A $p$ value of 0.05 or less was regarded as significant.

Five patients ( 3 males and 2 females) with a mean age of $30.2 \pm 12.2$ years were enrolled in this study (Table 1 ). The mean duration of the disease was $6.9 \pm 5.8$ years. All the subjects were receiving combination therapy with two or more antipsychotic agents and were administered benzodiazepine or antiparkinson drugs before dose-reduction (Table 1). The mean number of antipsychotic agents before dose-reduction was $2.4 \pm 0.9 \mathrm{mg}$. The mean total CP-converted dose was $695 \pm 97.5 \mathrm{mg}$. The mean total biperiden (BP)-converted and diazepam (DAP)-converted dose were $2.9 \pm 2.0 \mathrm{mg}$ and $7.9 \pm 6.8 \mathrm{mg}$, respectively. The use of antipsychotic agents after dose-reduction is shown in Table 1. The switch to monotherapy with an antipsychotic agent was achieved in all patients. The number of antiparkinson drugs, total BP-converted dose, number of benzodiazepine drugs, and total DAP-converted dose also decreased, but these differences were not significant.

There were no significant changes in the BPRS or DIEPSS scores after dose-reduction (Table 2). There was no change in the SAI-J score after dose-reduction (Table 2). Although there was an improvement in the DAI-10 score, it was not significant (Table 2). The results of subjective survey before and after dose-reduction are also
Table 2. Changes in psychiatric symptoms, adverse reactions, and satisfaction before and after dose-reduction

\begin{tabular}{|c|c|c|c|c|c|c|c|}
\hline \multirow{2}{*}{ Variable } & \multirow{2}{*}{$\begin{array}{l}\text { Dose- } \\
\text { reduction }\end{array}$} & \multicolumn{6}{|c|}{ Patient No. } \\
\hline & & 1 & 2 & 3 & 4 & 5 & Total \\
\hline \multirow[t]{2}{*}{ BPRS } & Before & 32 & 28 & 23 & 31 & 49 & $32.6 \pm 9.8$ \\
\hline & After & 20 & 43 & 25 & 41 & 45 & $34.8 \pm 11.5$ \\
\hline \multirow[t]{2}{*}{ DIEPSS } & Before & 0 & 5 & 4 & 5 & 3 & $3.4 \pm 2.1$ \\
\hline & After & 2 & 0 & 2 & 2 & 2 & $1.6 \pm 0.9$ \\
\hline \multirow[t]{2}{*}{ SAI-J } & Before & 20 & 14 & 14 & 11 & 15 & $14.8 \pm 3.3$ \\
\hline & After & 18 & 16 & 19 & 8 & 13 & $14.8 \pm 4.4$ \\
\hline \multirow[t]{2}{*}{ DAI-10 } & Before & 4 & 4 & -6 & 4 & 8 & $2.8 \pm 5.2$ \\
\hline & After & 8 & 2 & 8 & 2 & 10 & $6.0 \pm 3.7$ \\
\hline \multicolumn{8}{|c|}{ Self-assessment } \\
\hline \multirow{2}{*}{$\begin{array}{r}\text { Psychiatric } \\
\text { symptoms }\end{array}$} & Before & 1 & 3 & 1 & 1 & 3 & $1.8 \pm 1.1$ \\
\hline & After & 1 & 3 & 1 & 1 & 3 & $1.8 \pm 1.1$ \\
\hline \multirow{2}{*}{$\begin{array}{l}\text { Adverse } \\
\text { reactions }\end{array}$} & Before & 3 & 4 & 3 & 3 & 4 & $3.4 \pm 0.5$ \\
\hline & After & 1 & 1 & 3 & 1 & 1 & $1.4 \pm 0.9^{*}$ \\
\hline Satisfaction & After & 3 & 2 & 3 & 3 & 2 & $2.6 \pm 0.5$ \\
\hline
\end{tabular}

Values are presented as score only or mean \pm standard deviation. BPRS, Brief Psychiatric Rating Scale; DIEPSS, Drug-induced Extrapyramidal Symptoms Scale; SAI-J, Japanese version of the Schedule for Assessment of Insight; DAI-10, Drug Attitude Inventory-10 Questionnaire. ${ }^{*} p<0.05$ vs. before dose-reduction (paired $t$ test).

presented in Table 2. Before dose-reduction, 2 of the 5 patients complained of psychiatric symptoms. Regarding adverse reactions before dose-reduction, all patients complained of extrapyramidal and autonomic nervous symptoms. However, there was no deterioration in psychiatric symptoms, and 4 of the 5 patients reported a reduction in adverse reactions. Furthermore, 3 of the 5 patients were satisfied with the dose-reduction.

\section{DISCUSSION}

This is the first case report using both subjective and objective assessments of patients with schizophrenia and switching individuals from antipsychotic polypharmacy to monotherapy. The dose-reduction related changes in psychiatric symptoms were evaluated. No significant worsening of symptoms from the BPRS and the subjective survey was observed after one month of dose-reduction, suggesting that the SCAP method is useful.

The influence of dose-reduction on adverse reactions was also evaluated using the DIEPSS and subjective survey, which showed a reduction in adverse reactions in 4 of the 5 patients, improving the patients' quality of life. Our results are supported by a report in which adverse reactions were significantly decreased by reducing the 
number and doses of antipsychotic agents [3]. In the present study, physicians, pharmacists, and psychologists cooperated to perform drug adjustments at regular intervals. In all patients, the switch to monotherapy with an antipsychotic agent was achieved. However, the decrease in the total CP-converted dose was only 3\%. Yamanouchi et al. [9] reported that regular drug adjustments by attending physicians reduced the $\mathrm{CP}$-converted dose of antipsychotic agents from $1,012.3$ to $762.5 \mathrm{mg}$ (approximately a 25\% decrease). In our study, there were some discrepancies due to the following reasons: first, patients with a total CP-converted dose exceeding 1,000 mg before dose-reduction were not enrolled. Second, although monotherapy was achieved in Case 2, it was necessary to increase the total CP-converted dose by $240 \mathrm{mg}$ due to the deterioration of psychiatric symptoms. However, prescription proposal/drug adjustment intervention by pharmacists in cooperation with physicians/nurses may promote dose-reduction of antipsychotic agents, in light of the present results and the results presented by Shikata et al. [10].

In Japan, psychotropic polypharmacy is common for the treatment of schizophrenia. All subjects in this study were treated with concomitant psychotropic medications. The doses of antiparkinson drugs and benzodiazepines decreased with dose-reduction of antipsychotic agents. Although a relationship between adherence and polypharmacy has not yet been investigated in schizophrenia, these results indicate that adherence, as shown by DAI-10, increased after dose-reduction, suggesting a possible improvement in adherence.

No deterioration in psychiatric symptoms was observed in any patient. Of the 5 patients, 4 reported a reduction in adverse reactions and 3 were satisfied with the treatment. Patients not satisfied with their treatment may have been hoping to achieve rehabilitation and were not satisfied to have only their adverse reactions reduced. A study reported that participation in programs for social skills and neurocognitive individualized training $[11,12]$ as well as homecare or daycare use led to enhanced quality of life (QOL) and satisfaction in schizophrenic patients [13]. Therefore, to support rehabilitation, practitioners should encourage patients with schizophrenia to use these services.

In conclusion, the SCAP method is a safe and useful method that can be applied in a clinical setting. However, further examinations are required to determine why the patients were not satisfied with only reduction in adverse reactions. The use of social resources is important, and the improvement of the patient's QOL should be performed in cooperation with other fields.

\section{- Conflicts of Interest}

No potential conflict of interest relevant to this article was reported.

\section{- Author Contributions}

Conceptualization: Hiroyuki Kamei, Shigeki Yamada, Nakao Iwata. Data acquisition: Hanae Yamada, Masakazu Hatano, Manako Hanya, Hiroyuki Kamei. Formal analysis: Hanae Yamada, Masakazu Hatano, Manako Hanya, Hiroyuki Kamei. Supervision: Nakao Iwata. Writing-original draft: Hiroyuki Kamei. Writingreview \& editing: Nakao Iwata, Shigeki Yamada.

\section{ORCID}

Hiroyuki Kamei

https://orcid.org/0000-0003-3778-2352

Hanae Yamada https://orcid.org/0000-0002-2184-3870

Masakazu Hatano https://orcid.org/0000-0001-7032-878X

Manako Hanya https://orcid.org/0000-0003-2255-5157

Shigeki Yamada https://orcid.org/0000-0002-3891-1323

Nakao Iwata https://orcid.org/0000-0003-3189-6076

\section{REFERENCES}

1. Essock SM, Covell NH, Leckman-Westin E, Lieberman JA, Sederer LI, Kealey $\mathrm{E}$, et al. Identifying clinically questionable psychotropic prescribing practices for medicaid recipients in New York state. Psychiatr Serv 2009;60:1595-1602.

2. Correll CU, Kane JM. Is there a rationale for antipsychotic polypharmacy in schizophrenia? In: Fleischhacker WW, Hummer $M$, editors. Schizophrene Stoerungen-State of the Art III. Innsbruck: Verlag Integrative Psychiatrie;2004. p.95-112.

3. Essock SM, Schooler NR, Stroup TS, McEvoy JP, Rojas I, Jackson C, et al. Effectiveness of switching from antipsychotic polypharmacy to monotherapy. Am J Psychiatry 2011;168: 702-708.

4. Suzuki T, Uchida H, Tanaka KF, Nomura K, Takano H, Tanabe A, et al. Revising polypharmacy to a single antipsychotic regimen for patients with chronic schizophrenia. Int J Neuropsychopharmacol 2004;7:133-142.

5. Yoshio T, Inada T, Uno J, Miwa T, Kitagawa K, Miyahara Y, et al. Prescription profiles for pharmacological treatment of Japanese inpatients with schizophrenia: comparison between 2007 and 2009. Hum Psychopharmacol 2012;27:70-75.

6. Okumura Y, Noda T, Ito $\mathrm{H}$. [Antipsychotics prescribing pat- 
terns of patients with schizophrenia in Japan: using the national database of health insurance claim information and specified medical checkups]. Jpn J Clin Psychopharmacol 2013;16:1201-1215. Japanese.

7. Fleischhacker WW, Uchida H. Critical review of antipsychotic polypharmacy in the treatment of schizophrenia. Int J Neuropsychopharmacol 2014;17:1083-1093.

8. Sukegawa T, Inagaki A, Yamanouchi Y, Inada T, Yoshio T, Yoshimura R, et al. Study protocol: safety correction of high dose antipsychotic polypharmacy in Japan. BMC Psychiatry. 2014; 14:103.

9. Yamanouchi $Y$, Sukegawa T, Inagaki A, Inada T, Yoshio T, Yoshimura R, et al. Evaluation of the individual safe correction of antipsychotic agent polypharmacy in Japanese patients with chronic schizophrenia: validation of safe corrections for antipsychotic polypharmacy and the high-dose method. Int J
Neuropsychopharmacol 2014;18:pyu016.

10. Shikata Y, Iwanishi K, Tanaka S, Tensho M, Hashimoto Y. [Effect of inpatient pharmaceutical service for the doses on psychiatric drug therapy]. I Jpn Soc Hosp Pharm 2014;50: 579-584. Japanese.

11. Galderisi S, Piegari G, Mucci A, Acerra A, Luciano L, Rabasca $\mathrm{AF}$, et al. Social skills and neurocognitive individualized training in schizophrenia: comparison with structured leisure activities. Eur Arch Psychiatry Clin Neurosci 2010;260:305315.

12. Palumbo D, Mucci A, Piegari G, D'Alise V, Mazza A, Galderisi S. SoCIAL - training cognition in schizophrenia: a pilot study. Neuropsychiatr Dis Treat 2017;13:1947-1956.

13. Kao CC, Huang HM. A comparison of the quality of life of patients with schizophrenia in daycare and homecare settings. J Nurs Res 2014;22:126-135. 